\title{
A Chemomechanical Polymer that Functions in Blood Plasma with High Glucose Selectivity*
}

\author{
George K. Samoei, Weihua Wang, Jorge O. Escobedo, Xiangyang Xu, Hans-Jörg \\ Schneider, Robert L. Cook, and Robert M. Strongin \\ Prof. Dr. H.-J. Schneider, FR Organische Chemie, Universität des Saarlandes, 66041 Saarbrücken \\ (Germany), Fax: (+49)681-302-3209, E-mail:ch12hs@rz.uni-sb.de, G. K. Samoei, W. Wang, Dr. J. \\ O. Escobedo, X. Xu, Prof. R. L. Cook, Prof. R. M. Strongin, Department of Chemistry, Louisiana \\ State University, 232 Choppin Hall, Baton Rouge, LA 70803 (USA), Fax: (+1)225-578-3458, E-mail: \\ rstrong@lsu.edu
}

\section{Keywords}

biosensors; boronic acids; carbohydrates; chemomechanical system; glucose

The fabrication of reliable non- or minimally invasive glucose sensors, including those that embody automated insulin-delivery systems, has been a long-term goal of researchers from a variety of disciplines. The foremost challenge is the attainment of materials that are selective for glucose in bodily fluids, wherein a vast number of potential interferences exist.[1]

A number of chemomechanical polymers and related hydrogels have been investigated as potentially useful materials for glucose monitoring. Recent examples include glucose oxidase immobilized in hydrogels,[2] functionalized polymers coated on electrodes,[3] a crystalline colloidal array embedded in hydrogel networks,[4] and others.[5]

Few reports, however, describe fully synthetic hydrogel materials that change size in actual biological media in response to glucose. Asher et al. have described a boronic acid containing material that promotes the detection of glucose in tear solution.[4] Lowe and co-workers have reported glucose-induced size changes in a boronic acid functionalized polymer in cell-culture media.[6]

Herein we report a unique hydrogel which exhibits high glucose selectivity over other common blood sugars, such as fructose and galactose. It affords readily seen glucose-promoted size changes in human blood plasma. To the best of our knowledge, this is the first report of a chemomechanical polymer composed entirely of synthetic materials that exhibits a selective, rapid, and reversible size change in the presence of glucose in human blood plasma.

This effort is a start towards creating new materials that do not rely on biological materials for glucose-regulated drug delivery and, moreover, towards understanding new classes of chemomechanical polymers whose properties are highly tunable and amenable to an extensive degree of optimization. In this latter regard, the current study extends prior work, whereby flexible, selective supramolecular binding sites are introduced into pre-existing polymer networks in such a manner as to promote selective and easily seen macroscopic size changes. [7]

\footnotetext{
** We thank the National Institutes of Health for research support through award RO1 EB2044.

Correspondence to: Hans-Jörg Schneider; Robert M. Strongin.
} 
The chemomechanical polymer (A) is prepared (Scheme 1) by modifying commercial poly (methyl methacrylate) (PMMA; MW $350000 \mathrm{gmol}^{-1}$ ) with diethylenetriamine (1), dodecylamine (2), butylamine (3) and 3-aminophenyl-boronic acid (4).

Polymer $\mathbf{A}$ is characterized by elemental analysis, solid-state ${ }^{13} \mathrm{C}$ cross-polarization magic angle spinning (CP-MAS) NMR spectroscopy, and FTIR (see the Supporting Information). Polymer A is allowed to swell completely for several hours in water and is then cut into pieces of $8 \times 4 \times 1 \mathrm{~mm}^{3}$. The cut polymer is immersed in a solution of glucose $(0.005 \mathrm{M}$, approximating actual human physiological levels) and begins to contract immediately. A significant size change is observed over time (Figure 1). Polymer $(\mathbf{B})$ is prepared under conditions identical to those described for $\mathbf{A}$, but in the absence of 3-aminophenylboronic acid (4). No size change is observed for polymer $\mathbf{B}$ after immersion in glucose solution for $50 \mathrm{~min}$ (Figure 2). This indicates that the glucose-responsive behavior is due to the boronic acid moiety.

Polymer A also exhibits reversibility. The contracted polymer swells to its original size within two minutes after immersion in a $0.05 \mathrm{M} \mathrm{NaOH}$ solution. After it is removed from the $\mathrm{NaOH}$ solution and washed with distilled water, the swollen polymer contracts once more when immersed again in a $0.005 \mathrm{M}$ glucose solution (Figure 3).

Further studies also show that polymer $\mathbf{A}$ is selective for glucose over the other two common blood sugars, fructose and galactose (Figure 4). The error bars in Figure 4 indicate the reproducibility of the glucose-responsive contraction of polymer $\mathbf{A}$. Three different batches of polymer A were tested on three different days.

In a commercial sample of human blood plasma, similar glucose responsiveness and reproducibility are observed. A piece of swollen polymer $\mathbf{A}$ is immersed in a solution of human blood plasma that contains $0.005 \mathrm{M}$ added glucose. The polymer contracts sharply within 5$10 \mathrm{~min}$ (Figure 5). No response is observed for galactose and fructose (see the Supporting Information).

Bidentate binding of glucose by two boronic acid moieties is known to occur, in certain cases, selectively over galactose and fructose.[8] Shrinkage has been observed previously in other examples of glucose-induced cross-linkage of boronic acid containing polymers. [9] In our system, the glucose molecule also acts as an additional cross-link (besides the triamine). This results in the expulsion of water and gel shrinkage.

Additionally, sugar-binding-induced $\mathrm{H}^{+}$release has been used previously by Arnold et al. (for signal transduction) in a boronic acid containing hydrogel sensor for glucose.[10] In an analogous manner, we propose that the presence of emerging cationic charge from water and glucose bound to boron should stabilize the anionic nature of glucose-bound boron, thereby reducing the electrostatic repulsion and solvation by water that would lead to swelling. This may serve as an additional and/or alternative mechanism to glucose cross-linking through bidentate binding to boron.

Conversely, treatment with hydroxide deprotonates the amino groups on the polymer and also adds to boron. The concomitant increase in anionic charges enhances electrostatic repulsion and solvation in the hydrogel, causing it to swell very nearly to its initial size.[11]

Kataoka and co-workers have reported innovative strategies towards synthetic hydrogels that have been proposed as glucose detection/self-regulated insulin-delivery systems.[12,5a] Polymer sensitivity was heightened through rational design of the copolymer structure. In their material, as well as in most other boronic acid containing hydrogels reported to date, the hydrogels were prepared through the polymerization of boronic acid containing monomers. In contrast, our method for the preparation of glucose-responsive hydrogels involves simple 
modification of commercial PMMA to achieve glucose selectivity as well as a more-rapid equilibration time. Furthermore, the type of macroscopic response (shrinkage or expansion) and the selectivity to a certain stimulus of the hydrogels can be controlled by simply varying modifiers (Table 1).

When the polymer is modified with the long-alkyl-chain amine, dodecylamine (modifier compound 2, Scheme 1), it exhibits expansion in all three sugar solutions (Table 1, entries 1 and 2). This may be attributed to steric hindrance of the alkyl chain towards bidentate binding of glucose by two boronic acid moieties. Thus, the binding of sugar molecules enhances the formation of charge on boron atoms and increases the polarity and water content of the polymer, resulting in polymer expansion rather than contraction.

In the case of butylamine (modifier compound $\mathbf{3}$, Scheme 1), the smaller butyl chain apparently does not significantly hinder bidentate binding of glucose. The cross-linking of polymer chains by glucose molecules can thereby overcome the effect of charge accumulation (see above), resulting in gel contraction (Table 1, entry 3). Furthermore, when a mixture of dodecylamine and butylamine is used (with half the original amounts each), as in polymer A (Table 1, entry 4), the gel contracts but to a lesser extent than when incorporating butylamine alone. This affords additional evidence that dodecyl sterically hinders cross-linking and contraction.

A significant difference in size change is observed when the length of the cross-linker is varied (Table 1, entries 1 and 2). In the presence of the same alkyl amine (dodecyl), the hydrogel with the longer-carbon-chain cross-linker (modifier 5, Scheme 1) exhibits a greater degree of expansion as compared with the case in which the shorter-carbon-chain cross-linker (1) is used. This is attributed to the fact that longer-chain cross-linkers provide wider cavities for the uptake of water.

The optimized polymer $\mathbf{C}$ (Table 1, entry 3 ) exhibits the highest degree of selective contraction in the presence of glucose. Importantly, we find that the swelling ratio of polymer $\mathbf{C}$ exhibits a linear decrease proportional to increasing glucose concentrations (Figure 6).

Figure 7 shows that polymer $\mathbf{C}$ serves as a "chemical corkscrew". After contraction triggered by glucose, release of the compound inside the capillary occurs (green ink solution for visualization). This methodology may thus promote the eventual optimization of selfregulating actuators that can work without interfacing to external devices.[7]

In summary, the general synthetic procedure developed[7a] for chemomechanical polymers is highly useful as the recognition groups can be readily introduced and the properties of the polymer can be easily optimized.[7] Current efforts involve further optimization of hydrogel properties through side-chain modification and miniaturization, which is known to enhance the performance of related materials. [7] Many challenges besides achieving glucose selectivity remain before a self-regulated insulin-delivery system can be implemented.[1] These include controlling potential interactions of the deliverable drug with the polymer,[13] response time, biocompatibility, calibration, and the material3s lifetime.

\section{Supplementary Material}

Refer to Web version on PubMed Central for supplementary material.

\section{References}

1. For a recent review and example, see: a) Arnold MA, Small GW. Anal Chem 2005;77:5429. [PubMed: 16131049] b) Beyer U, Fleischer A, Kage A, Haueter U, Ehwald R. Biosens Bioelectron 2003;18:1391. [PubMed: 12896841] and references therein 
2. This is a pH-responsive material: Kim D, Seo K, Park K. J Biomater Dent 2000;16:189.

3. Gabai R, Sallacan N, Chegel V, Bourenko T, Katz E, Willner I. J Phys Chem B 2001;105:8196.

4. Asher SA, Alexeev VA, Sharma AC, Goponenko AV, Das S, Lednev IK, Wilcox CS, Finegold DN. J Am Chem Soc 2003;125:3322. [PubMed: 12630888]

5. For an example, see: a) Kataoka K, Miyazaki H, Bunya M, Okano T, Sakurai Y. J Am Chem Soc 1998;120:12694. b) Cai Q, Zeng K, Ruan C, Desai TA, Grimes CA. Anal Chem 2004;76:4038.

[PubMed: 15253640] c) Suri JT, Cordes DB, Cappuccio FE, Wessling RA, Singaram B. Angew Chem 2003;115:6037.Angew Chem Int Ed 2003;42:5857. d) Kokufata E, Zhang YQ, Tanaka T. Nature 1991;351:302. e) Miyata T, Asami N, Uragami T. Nature 1999;399:766. [PubMed: 10391240]

6. Lee MC, Kabilian S, Hussain A, Yang X, Blyth J, Lowe CR. Anal Chem 2004;76:5748. [PubMed: 15456294]

7. a) Schneider HJ, Tianjun L, Lomadze N. Eur J Org Chem 2006;26:677. b) Lomadze N, Schneider HJ. Tetrahedron 2005;61:8694.and references therein

8. James TD, Sandanayake KRAS, Shinkai S. Angew Chem 1994;106:2207.Angew Chem Int Ed Engl 1994;33:2287.

9. Alexeev VL, Das S, Finegold DN, Asher SA. Clin Chem 2004;50:2353. [PubMed: 15459093]

10. For an example, see: Arnold FH, Zheng WG, Michael AS. J Membr Sci 2000;167:227.

11. Karadag E, Saraydin D. Turk J Chem 2001;26:863.

12. a) Matsumoto A, Ikeda S, Harada A, Kataoka K. Biomacromolecules 2003;4:1410. [PubMed: 12959613] b) Matsumoto A, Yoshida R, Kataoka K. Biomacromolecules 2004;5:1038. [PubMed: 15132698]

13. a) Nakamura K, Murray RJ, Joseph JI, Peppas NA, Morishita M, Lowman AM. J Controlled Release 2004;95:589. b) Morishita M, Goto T, Peppas NA, Joseph JI, Torjman MC, Munsick C, Nakamura K, Yamagata T, Takayama K, Lowman AM. J Controlled Release 2004;97:115. 

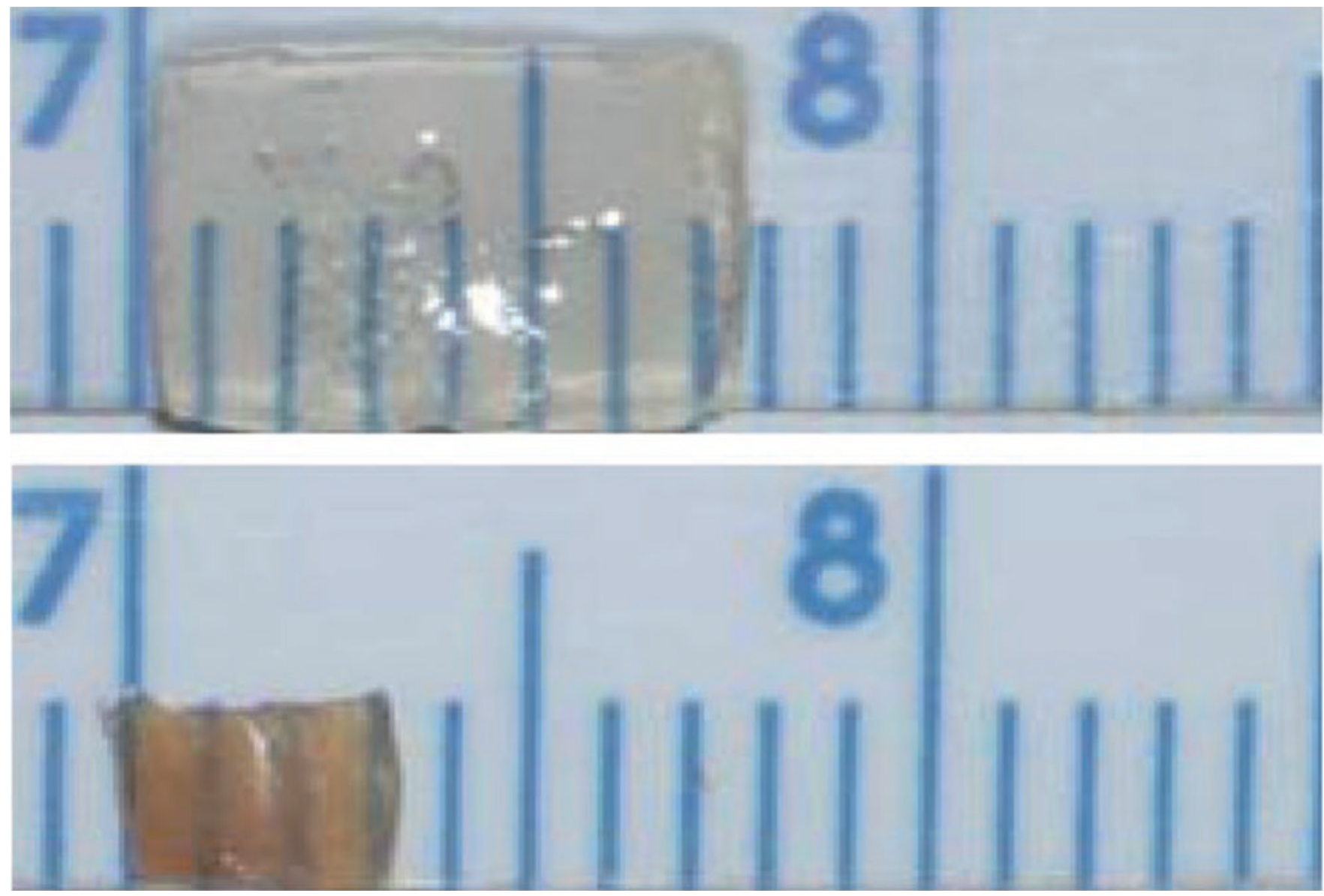

Figure 1.

Behavior of polymer $\mathbf{A}$ in response to glucose. Top: original sample, bottom: after $50 \mathrm{~min}$ in a $0.005 \mathrm{M}$ glucose solution. 

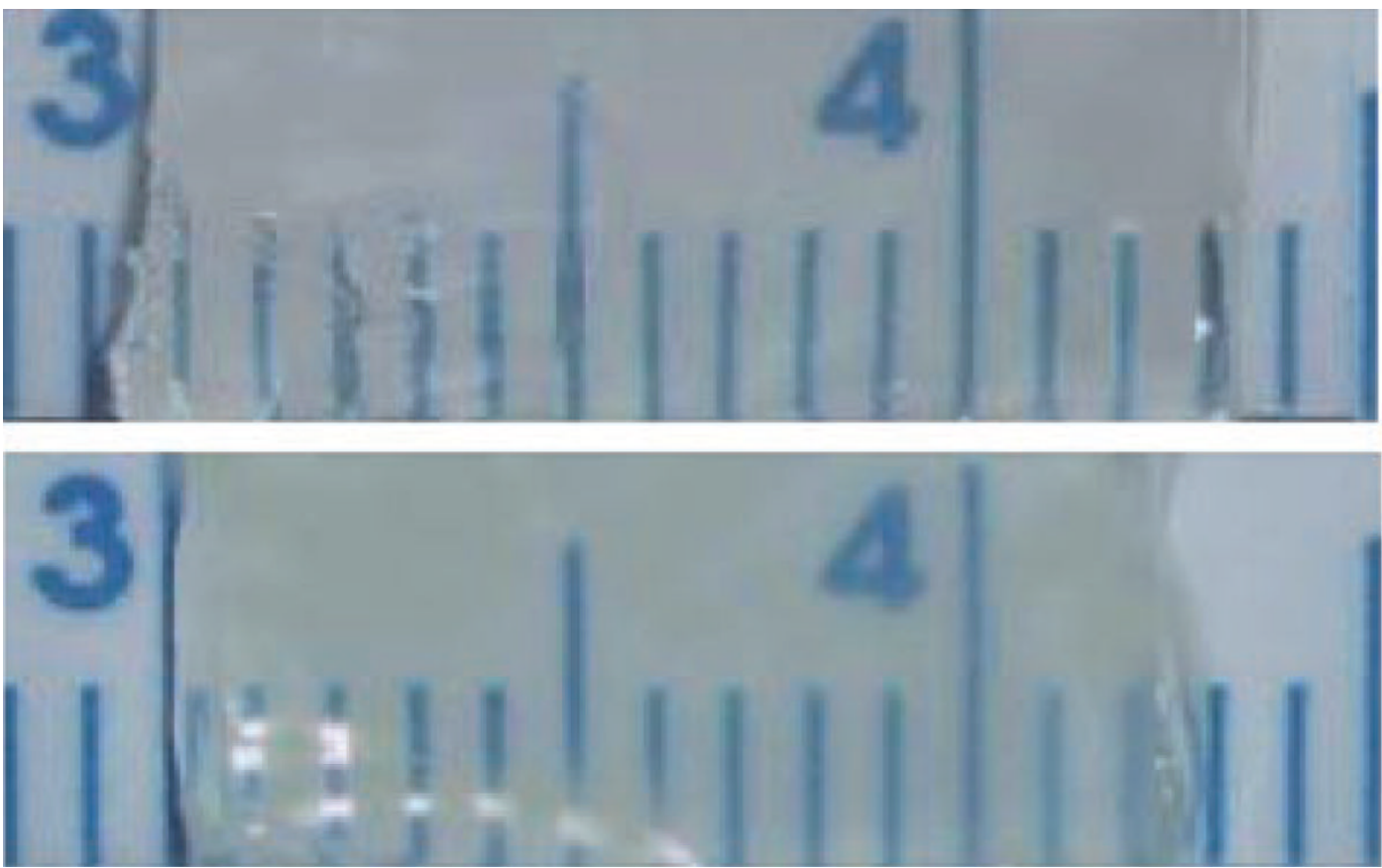

Figure 2.

Behavior of polymer $\mathbf{B}$ in response to glucose. Top: original sample, bottom: after $50 \mathrm{~min}$ in a $0.005 \mathrm{M}$ glucose solution. 


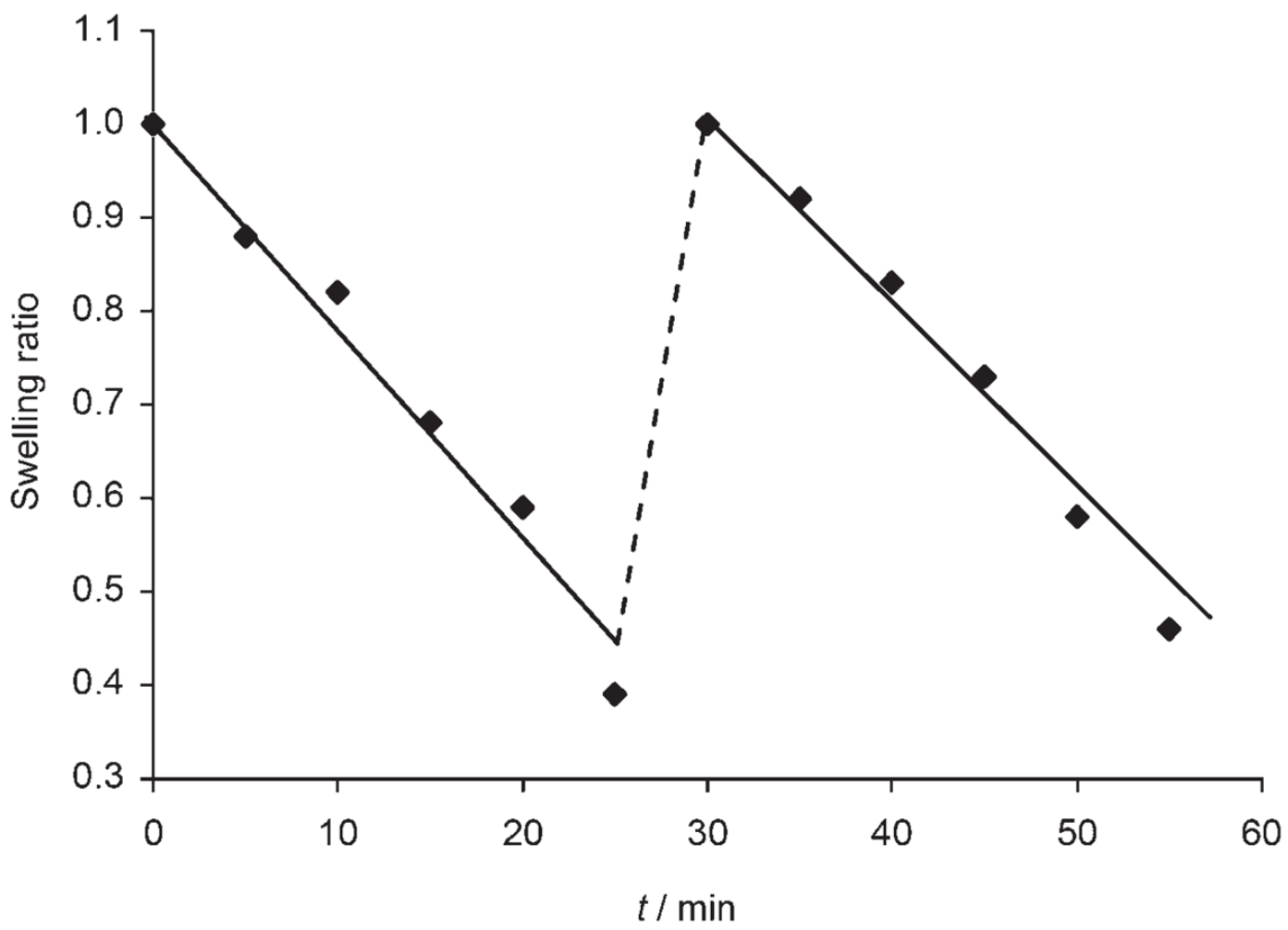

Figure 3.

Contraction-expansion of polymer $\mathbf{A}$ in response to glucose $(0.005 \mathrm{M})$. The polymer was expanded to its initial size between runs by rinsing with $0.05 \mathrm{M} \mathrm{NaOH}$ (dashed line). The size of the cut polymer after swelling in water in this experiment is $5 \times 5 \times 1 \mathrm{~mm}^{3}$. 


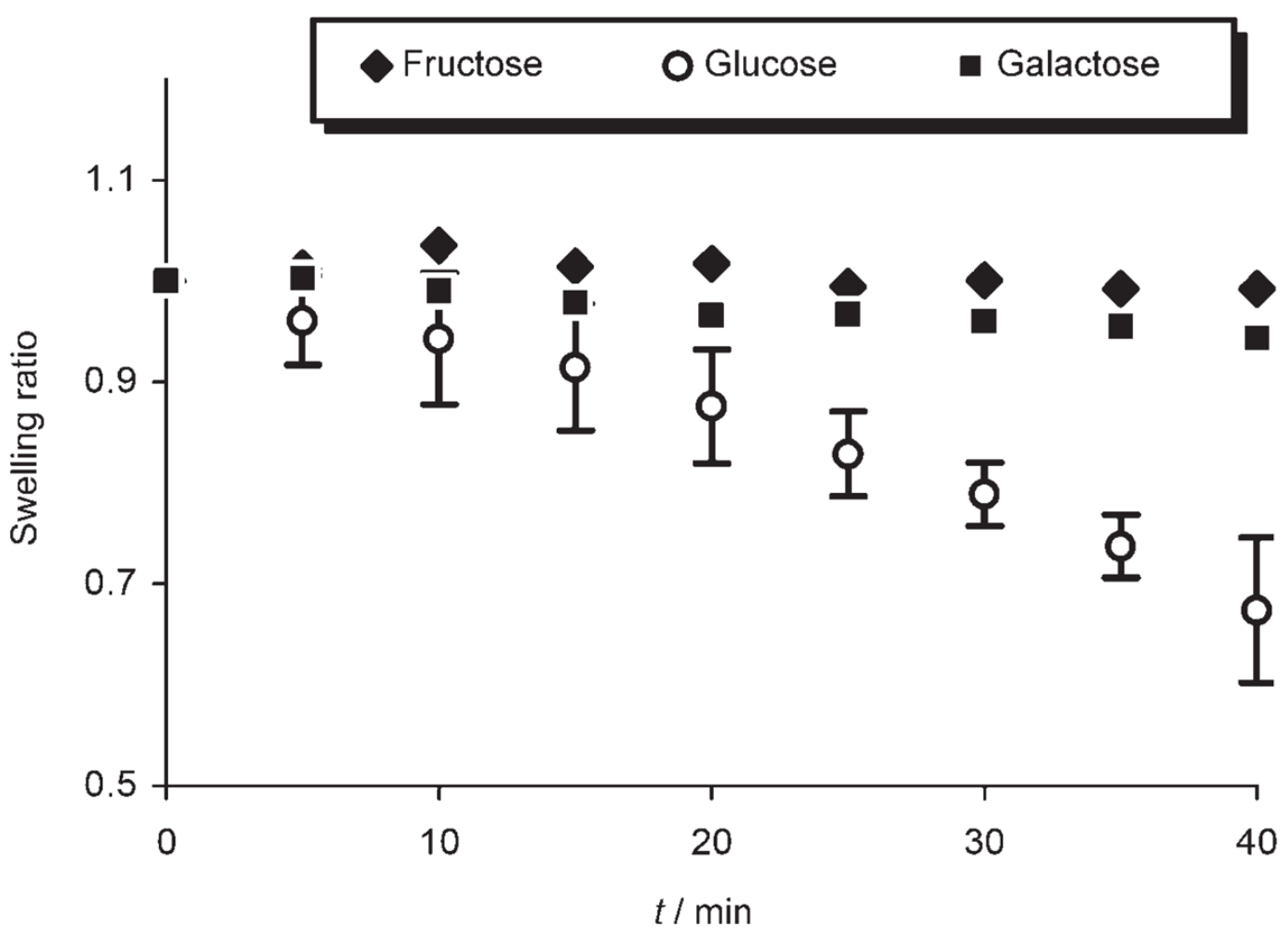

Figure 4.

Selective response of polymer $\mathbf{A}$ to glucose over fructose and galactose. All the three sugars are at the same concentration $(0.005 \mathrm{M})$. The size of the cut polymer after swelling in water in this experiment is $8 \times 4 \times 1 \mathrm{~mm}^{3}$. 


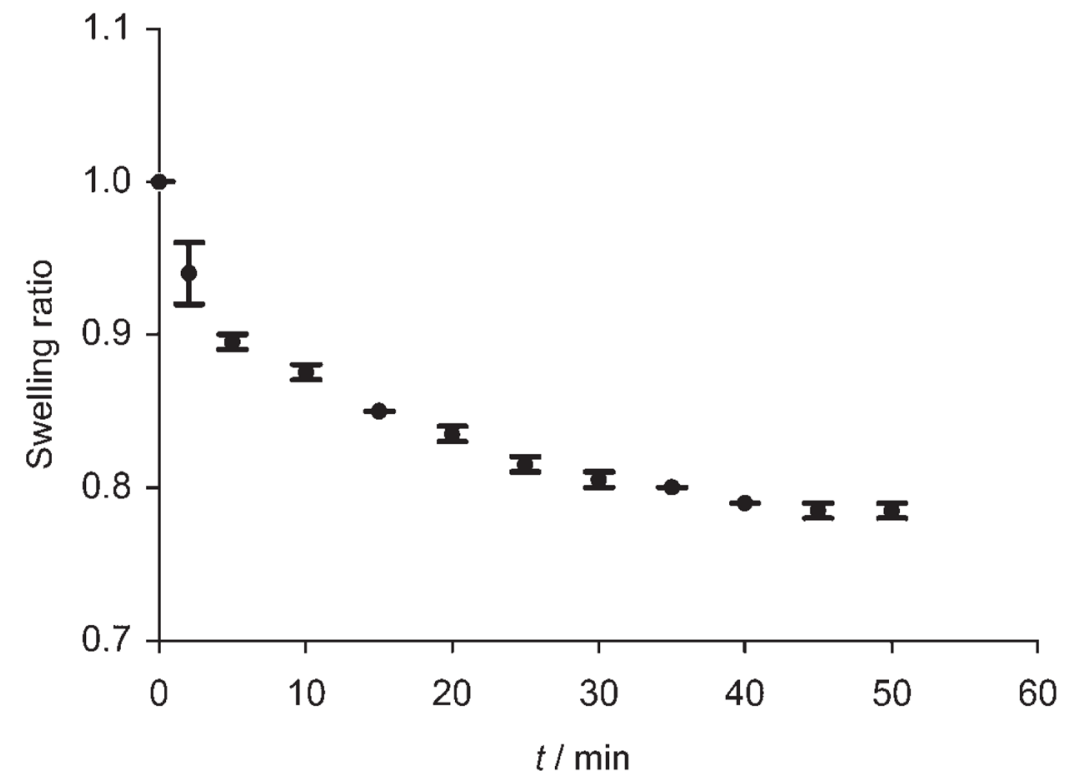

Figure 5.

Contraction of polymer $\mathbf{A}$ in the presence of reconstituted human plasma containing $0.005 \mathrm{M}$ glucose. The size of the cut polymer after swelling in water in this experiment is $8 \times 7 \times 1$ $\mathrm{mm}^{3}$. 


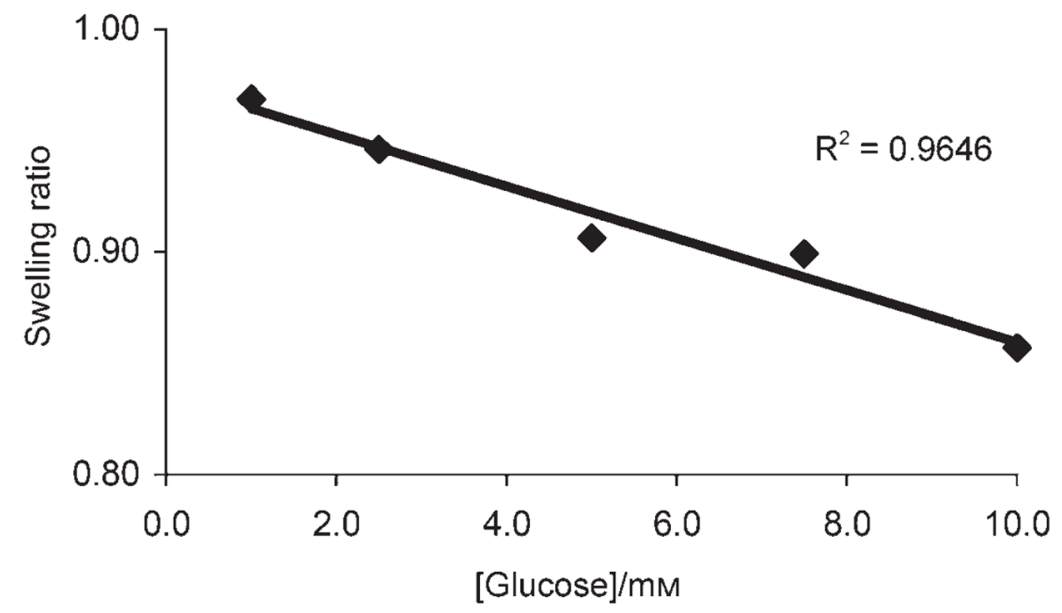

Figure 6.

Swelling ratio of polymer $\mathbf{C}$ in the presence of various concentrations of glucose at $25^{\circ} \mathrm{C}$. The values are the average of three consecutive determinations after $20 \mathrm{~min}$. The size of the cut polymer after swelling in water in this experiment is $14 \times 7 \times 1 \mathrm{~mm}^{3}$. 


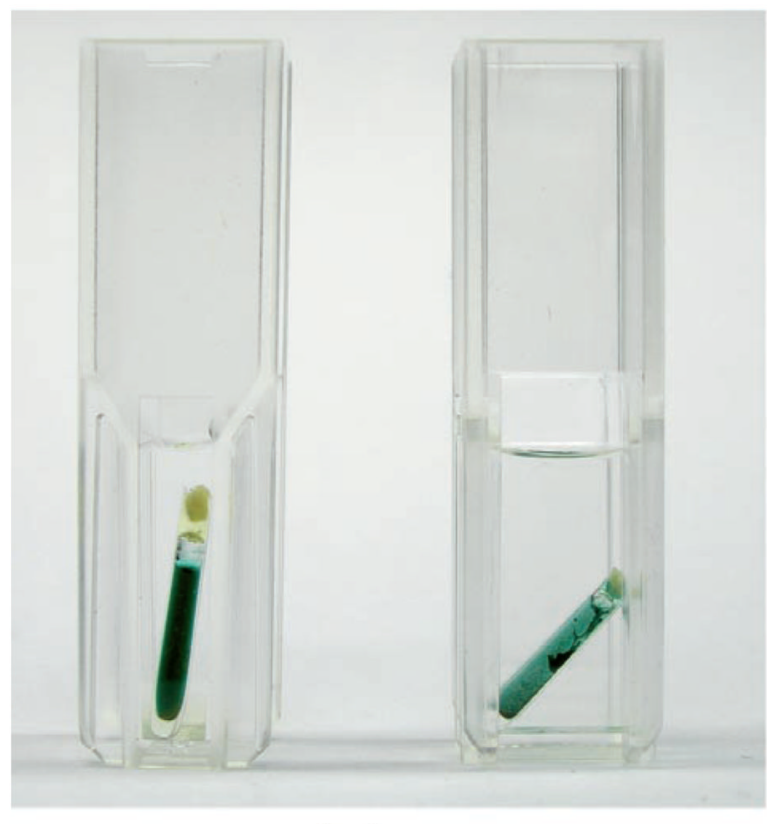

$0 \mathrm{~min}$

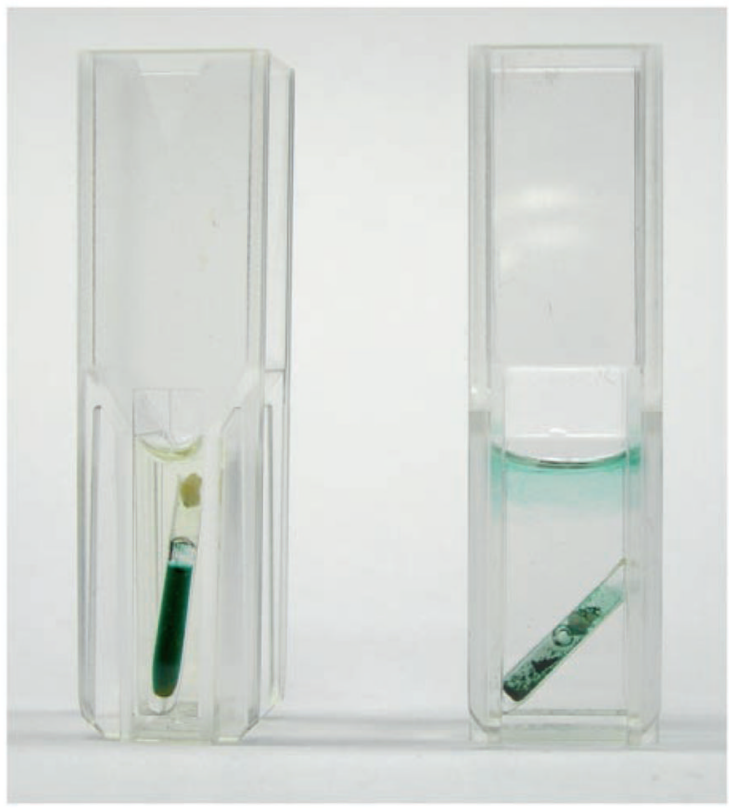

$25 \min$

Figure 7.

A demonstration with polymer $\mathbf{C}$ in glucose-regulated chemical delivery. A capillary tube is filled with ink and sealed with swollen polymer $\mathbf{C}$. The capillary is immersed in $\mathrm{H}_{2} \mathrm{O}$ (control, cuvette on the left in each picture) and in $0.005 \mathrm{M}$ glucose solution. After $25 \mathrm{~min}$, the ink is released only in the glucose solution owing to the contraction of the polymer (right). 


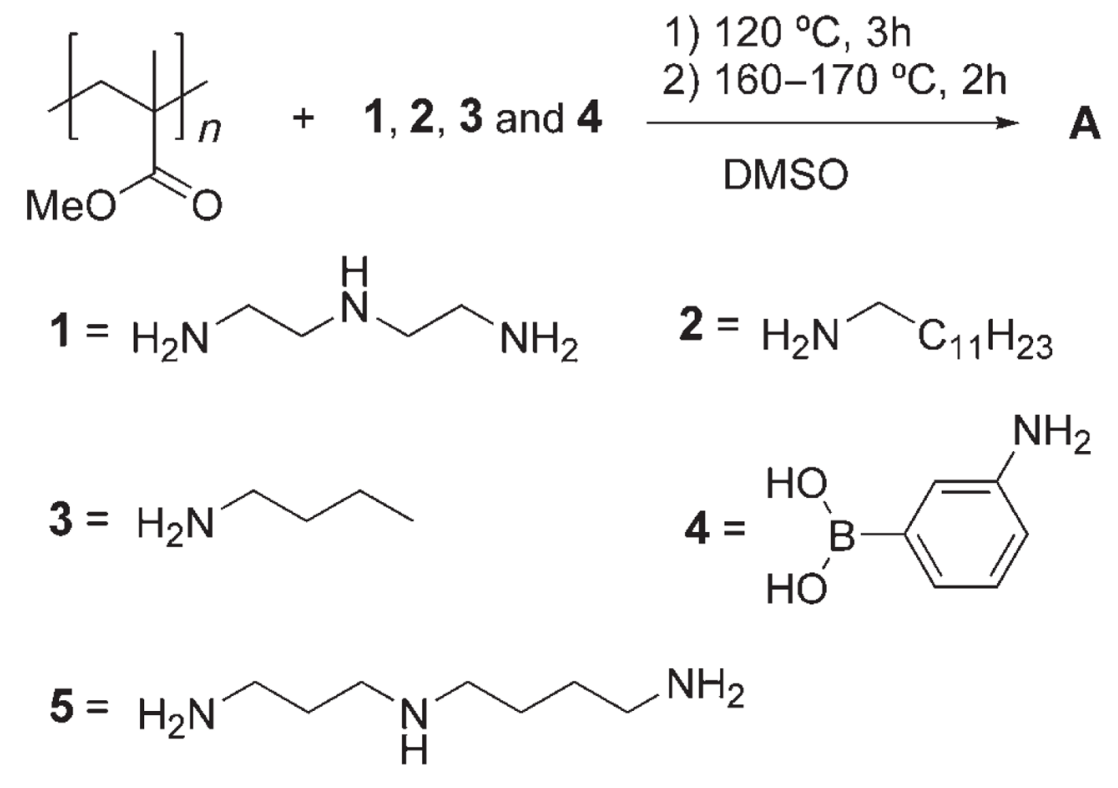

Scheme 1.

One-pot synthesis of polymer A. DMSO=dimethyl sulfoxide. 
Table 1

Response (shrinkage or expansion) and selectivity of the hydrogels to a certain stimulus. ${ }^{[a]}$

\begin{tabular}{|c|c|c|c|c|}
\hline Entry & Polymer modifiers & glucose $(0.005 \mathrm{M})$ & $\begin{array}{l}\text { Swelling ratio } \\
\text { galactose } \\
(0.005 \mathrm{M})\end{array}$ & fructose $(0.005 \mathrm{M})$ \\
\hline 1 & $5+2+4$ & 1.70 & 1.61 & 1.50 \\
\hline 2 & $1+2+4$ & 1.22 & 1.25 & 1.31 \\
\hline 3 & $1+3+4$ & 0.63 & 0.96 & 0.93 \\
\hline 4 & $1+2+3+4$ & 0.83 & 0.99 & 0.97 \\
\hline
\end{tabular}

${ }^{[a]}$ The size of the cut polymers is $8 \times 4 \times 1 \mathrm{~mm}^{3}$. Swelling ratios are obtained after $25 \mathrm{~min}$. 